\title{
TRATAMENTO ORTO-CIRÚRGICO DE LATERO-DESVIO MANDIBULAR:
} RELATO DE CASO

\section{ORTHO-SURGICAL TREATMENT OF MANDIBULAR LATERAL DEVIATION:}

\section{CASE REPORT}

\section{Ian Magalhães de Andrade}

Residente em Cirurgia Buco Maxilo Faciais do Hospital Federal dos Servidores do Estado do Rio de Janeiro - HFSE.

\section{Anderson das Mercês}

Aluno de Graduação da Faculdade de Odontologia da Universidade Iguaçu - UNIG

\section{Marcelo José Uzeda}

Professor da Disciplina de Cirurgia Bucal da Universidade Federal Fluminense - UFF e Professor das disciplinas de Cirurgia Bucal, Semiologia e Anatomia da Faculdade de Odontologia da Universidade Iguaçu - UNIG.

\section{Rodrigo Resende}

Professor da Disciplina de Cirurgia Bucal da Universidade Federal Fluminense - UFF e Professor das disciplinas de Cirurgia Bucal e Semiologia da Faculdade de Odontologia da Universidade Iguaçu - UNIG.

\section{Suelen Cristina Sartoretto}

Professora das disciplinas de Cirurgia Bucal, Semiologia e Anatomia da Faculdade de Odontologia da Universidade Iguaçu - UNIG.

Instituição: Hospital Federal dos Servidores do Estado do Rio de Janeiro/RJ

Categoria: Relato de caso

Informações do autor principal: Ian Magalhães de Andrade. Estrada da Água Branca n 4970, Bangu, CEP 21862-371, Rio de Janeiro-RJ. ian_mag@ hotmail.com 


\title{
RESUMO
}

A assimetria facial é uma anomalia que ocorre entre partes homólogas da face, que pode gerar diversas alterações na harmonia facial e problemas funcionais, como dificuldade mastigatória. Essas alterações podem causar grandes prejuízos psicossociais ao indivíduo que possui esse tipo de anormalidade dentofacial. Dentre as opções terapêuticas, a que mais se destaca é a cirurgia ortognática pois produz resultados previsíveis e com boa estabilidade a longo prazo. O objetivo do presente trabalho é relatar o caso clínico de uma paciente portadora de látero-desvio mandibular e importante assimetria facial, apresentando o resultado alcançado com a cirurgia ortognática bimaxilar e discutir a literatura especializada.

Palavras-chave: Cirurgia ortognática; Látero-desvio mandibular; Ortodontia.

\begin{abstract}
Facial asymmetry is an abnormality that occurs between homologous parts of the face, which can generate several changes in facial harmony and functional problems, as masticatory difficulty. These changes can cause great psychosocial damage to the individual who has this type of dentofacial abnormality. Among the therapeutic options, the most common is orthognathic surgery, because produces predictable results with good long-term stability. The aim of the present study is to report the clinical case of a patient with mandibular lateral deviation and an important facial asymmetry, presenting the result achieved with bimaxillary orthognathic surgery and discuss the specialized literature.
\end{abstract}

Key words: Ortognatic surgery; Mandibular lateral deviation; Orthodontics 


\section{INTRODUÇÃO}

Assimetria faciai pode ser definida como um desequilíbrio que ocorrem entre partes homólogas da face, afetando as proporções dessas partes entre si em relação ao tamanho, forma e posição. A assimetria do complexo dentofacial pode ser tanto unilateral quanto bilateral, podendo ocorrer nas seguintes direções: Antero-posterior, Superoinferior e Medio-lateral (FISCHER, B., 1956). Essa condição afeta aproximadamente $20 \%$ da população brasileira, podendo os pacientes com estas discrepâncias apresentar vários graus de comprometimento estético e funcional (SILVA, E.D.O., 2004).

Com relação a sua etiologia, as assimetrias mandibulares podem ocorrer devido a uma alteração do crescimento (excessivo ou deficiente) do corpo e ramo mandibulares, ou a mandíbula pode estar desviada em função de um crescimento assimétrico de outras estruturas anatômicas (MEDEIROS, J.P., MEDEIROS P.P., 2004). Geralmente essas assimetrias mandibulares podem ser caracterizadas clinicamente por: desvio do mento para um lado, discrepância dento-esqueletal da linha média, mordida cruzada e discrepância no sentido vertical da mandíbula (SKOLNICK, J., 1994).

Em pacientes que possuem como agente etiológico o componente esquelético não associado a qualquer patologia, a abordagem cirúrgica é o tratamento de escolha, sendo a cirurgia ortognática uma das técnicas mais utilizadas na correção das assimetrias mandibulares (Araújo, A., 1999). Em algumas situações, o tratamento orto-cirúrgico pode associar uma cirurgia combinada de maxila e mandíbula. Vários fatores podem indicar a necessidade para a realização da cirurgia ortognática, entretanto considera-se uma das mais importantes o efeito psicossocial que tal anormalidade pode ocasionar (Silva, E.D.O., 2004).

O objetivo desse trabalho é apresentar um caso de correção de assimetria facial com cirurgia ortognática, apresentando os resultados alcançados pela técnica cirúrgica e discutindo a literatura especializada.

\section{CONSIDERAÇÕES ÉTICAS}

Os procedimentos empregados seguiram os padrões éticos da Declaração de Helsinque de 1975, revisada em 2000. O paciente foi submetido a tratamento cirúrgico 
no Hospital Federal dos Servidores do Estado do Rio de Janeiro conveniado com a Universidade Federal Fluminense através da Residência em Cirurgia e Traumatologia Buco Maxilo Faciais, após assinar o termo de consentimento livre e esclarecido para uso de imagens e dados para publicação científica. $\mathrm{O}$ estudo foi conduzido de acordo com as Diretrizes do Relatório de Caso Clínico (CARE, www.care-statement.org).

\section{CASO CLÍNICO}

Paciente sexo feminino, 33 anos, melanoderma, apresentava queixa principal de dificuldade mastigatória associada a estética facial deficiente. Ao exame clínico foi constatado uma assimetria mandibular (látero-desvio à esquerda) sem alterações no sentido antero-posterior.

Foi solicitado uma cintilografia da articulação temporomandibular com o objetivo de avaliar distúrbios no crescimento ósseo do côndilo mandibular. Após a realização do exame, não foi observado nenhuma alteração digna de nota

Após exame clínico, foram obtidos exames de imagem como radiografias cefalométrica de perfil e panorâmica para planejamento do caso. $\mathrm{Na}$ análise cefalométrica, observou-se os seguintes valores: $\mathrm{SNA}=87^{\circ}, \mathrm{SNB}=83^{\circ}$, Plano Oclusal $=20^{\circ}$ e Plano Mandibular $=44^{\circ}$.

No protocolo fotográfico (Figura 1 - A, B, C) podemos observar um látero-desvio mandibular à esquerda exuberante, como observado no exame clínico.
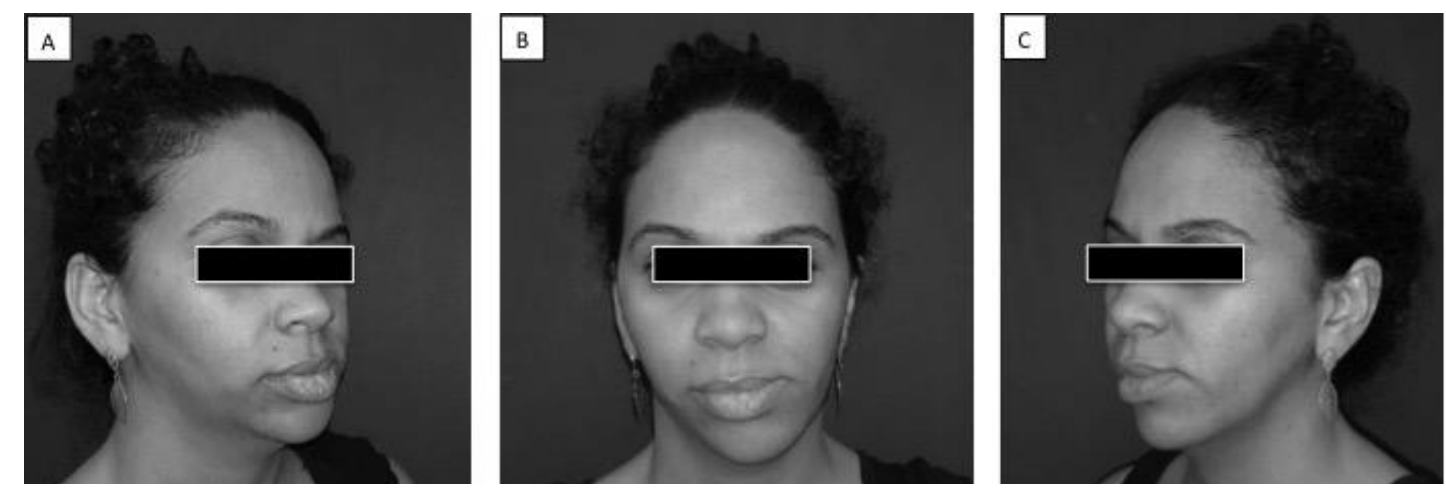

Figura 1: aquisição de imagens pré-operatórias evidenciando o látero-desvio mandibular 


\section{Plano de tratamento e tratamento ortodôntico}

Após o diagnóstico de assimetria mandibular (látero-desvio à esquerda), a paciente foi encaminhada a um serviço de ortodontia para realizar o tratamento pré-operatório. Os objetivos da instalação do aparelho fixo ortodôntico foram alinhar e nivelar os elementos dentários e reposicionar os dentes nas respectivas bases ósseas.

Foram obtidos modelos de estudo ao longo do tratamento para verificação da oclusão. Após a obtenção das movimentações desejadas, a cirurgia de modelos foi realizada para complementação do diagnóstico e confecção das guias cirúrgicos transoperatórios.

\section{Cirurgia ortognática e pós-operatório}

O procedimento cirúrgico foi realizado sob anestesia geral no Hospital Federal dos Servidores do Estado do Rio de Janeiro. Os movimentos selecionados para o caso foram obtidos durante a cirurgia de modelo, sendo eles: Avanço maxilar de 2,5mm com reposição inferior dos molares de $3 \mathrm{~mm}$ e incisivos $1 \mathrm{~mm}$; Avanço mandibular de 2mm; Correção do cant de $5 \mathrm{~mm}$ que era inferior à direita.

Após acessos cirúrgicos intraorais, foram realizadas as osteotomias que possibilitaram a realização dos movimentos planejados durante a cirurgia de modelo, realizando a fixação dos ossos gnáticos com miniplacas e parafusos.

A paciente apresentou boa evolução pós-operatória (Figura $2-\mathbf{A}, \mathbf{B}$, C), levando a um resultado de expressiva melhora no perfil facial da paciente, impactando diretamente nas questões funcionais e estéticas da paciente (Figura 3-A, B).
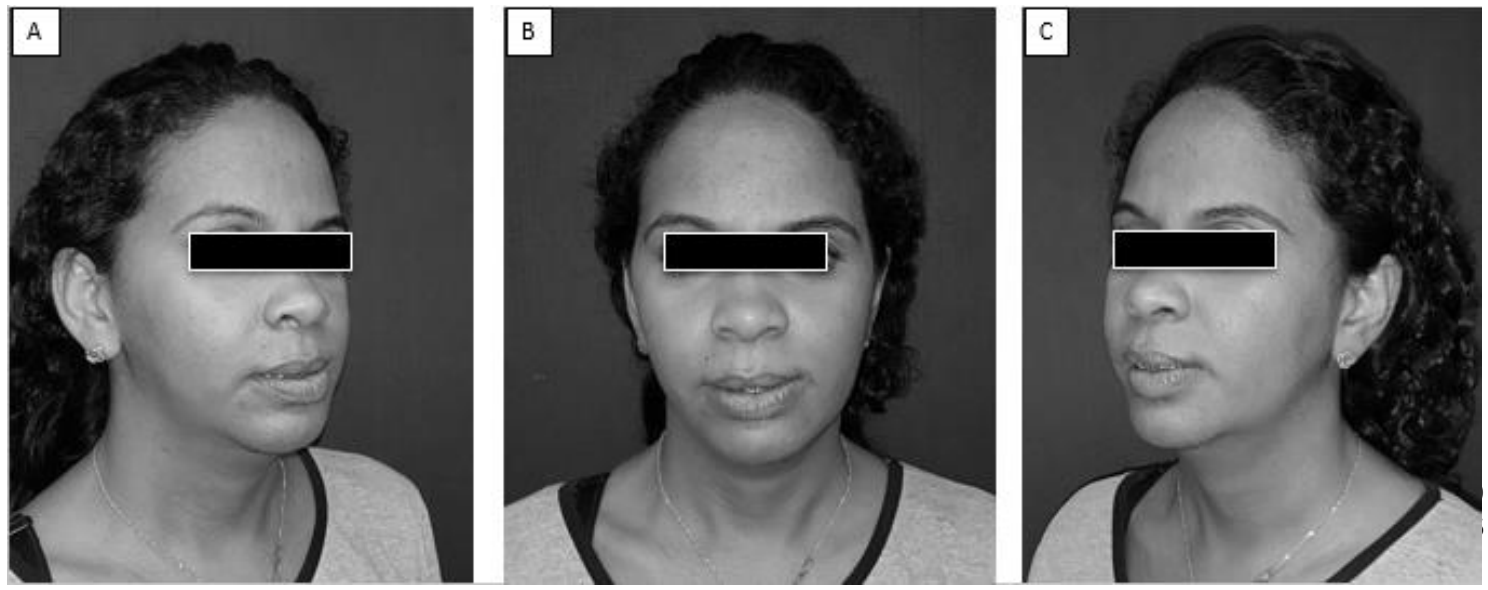


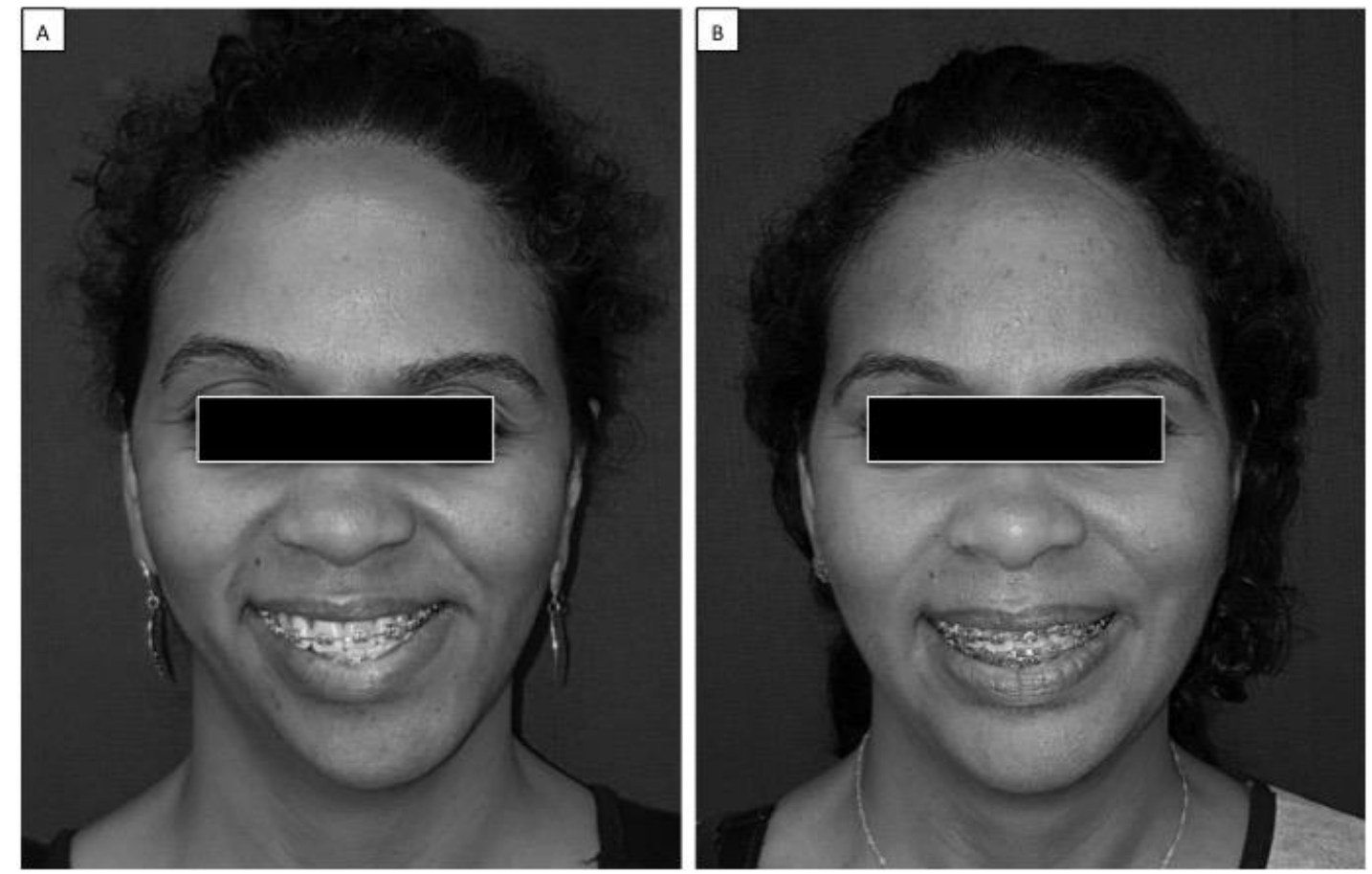

Figura 3: Imagem comparativa do pré-operatório (A) com o Pós-Operatório (B)

\section{DISCUSSÃO}

A simetria facial é raramente encontrada, devido a diversos fatores que podem gerar diferentes graus de assimetrias que são leves e imperceptíveis (THIESEN, N.G., GRIBEL, B.F., FREITAS, M.P.M., 2015). No entanto, quando as assimetrias são exacerbadas, podem levar a alterações estéticas e problemas funcionais no desenvolvimento orofacial, nutricional e psicossocial nos pacientes (SRIVASTAVA, D., 2018). 
Para correção de tal condição, a cirurgia ortognática é uma das opções terapêuticas mais utilizadas, quando o agente etiológico é o componente ósseo sem a presença de outras patologias envolvidas no processo (ARAÚJO, A., 1999).

Silva E.D.O., 2004, realizaram um relato de caso, semelhante ao aqui discutindo, em que o paciente tratado com cirurgia ortognática apresentava um látero-desvio mandibular para a esquerda com alterações no sentido antero-posterior (deformidade dentofacial do tipo classe III). Em sua conclusão, o autor confirma que a cirurgia ortognática é eficiente devido ao resultado previsível, a estabilidade alcançada a longo prazo e pôr sem bem estudada e revisada na literatura.

Marlière, D.A.A., 2018, realizaram um trabalho com o objetivo de descrever e comparar diferentes métodos de planejamento em cirurgia ortognática (convenciona e virtual) e seus resultados no pós-operatório no tratamento de pacientes com assimetria facial. Eles concluem o trabalho que todos os métodos de planejamento (convencional e virtual) demonstraram previsibilidade e foram viáveis em pacientes assimétricos, proporcionando resultados satisfatórios, como o resultado obtido por Silva, E.D.O., 2004.

\section{CONCLUSÃO}

Diante do caso exposto, concluímos que as assimetrias faciais, como o láterodesvio mandibular, exercem grande influência na função mastigatória e em uma estética facial deficiente e que a cirurgia ortognática se classifica como a melhor opção terapêutica para esses casos devido sua capacidade de corrigir as principais consequências causada pela assimetria, impactando de forma direta no efeito psicossocial que essa condição pode proporcionar.

\section{REFERÊNCIAS BIBLIOGRÁFICAS:}

1. Araújo, A. Cirurgia Ortognática. 1a ed. São Paulo: Livraria Santos Editora, 1999, $374 p$

2. Fischer, B. Asymmetries of the dentofacial complex. Angle Orthod.1954;24(4):17992.

3. Marlière, D.A.A et al. Comparação de diferentes planejamentos em cirurgia ortognática para tratamento de assimetrias faciais: relato de casos. HU Revista, v. 44, n. 1, p. $131-141,8$ fev. 2019. 
4. Medeiros, J.P.; Medeiros, P.P. Cirurgia ortognática para o ortodontista. 2nd ed. São Paulo: Ed. Santos; 2004.

5. Silva E.D.O et al. Tratamento cirúrgico de assimetria mandibular: relato de caso clínico. Rev Cir Traumat Buco-Maxilo-Fac. 2004;4(1):23-9.

6. Skolnick, J. et al. Prepubertal trauma and mandibular asymmetry in orthognathic surgery and orthodontic patients. Am J Orthod DentofacialOrthop; 105(1): 73-7, 1994 Jan.

7. Srivastava, D. et al. Facial asymmetry revisited: part I- diagnosis and treatment planning. Journal of Oral Biology and Craniofacial Research, v. 8, n. 1, p. 7-14, apr. 2018.

8. Thiesen N.G.; Gribel B.F.; Freitas M.P.M. Facial asymmetry: a current review. Dental Press Journal of Orthodontics, v. 20, n. 6, p. 110-125, dec. 2015. 